\title{
Optimized Hole Injection with Strong Electron Acceptors at Organic-Metal Interfaces
}

\author{
Norbert Koch, ${ }^{*}$ Steffen Duhm, and Jürgen P. Rabe \\ Institut für Physik, Humboldt-Universität zu Berlin, Newtonstrasse 15, D-12489 Berlin, Germany
}

Antje Vollmer

Berliner Elektronenspeicherring-Gesellschaft für Synchrotronstrahlung mbH, D-12489 Berlin, Germany

Robert L. Johnson

Institut für Experimentalphysik, Universität Hamburg, Luruper Chaussee 149, D-22761 Hamburg, Germany

(Received 17 June 2005; published 30 November 2005)

\begin{abstract}
The energy-level alignment at interfaces between three electroactive conjugated organic materials and $\mathrm{Au}$ was systematically varied by adjusting the precoverage of the metal substrate with the electron acceptor tetrafluoro-tetracyanoquinodimethane (F4-TCNQ). Photoelectron spectroscopy revealed that electron transfer from Au to adsorbed F4-TCNQ was responsible for lowering the hole-injection barrier by as much as $1.2 \mathrm{eV}$. This novel interface modification scheme is independent of the charge transfer complex formation ability of the organic materials with the electron acceptor.
\end{abstract}

DOI: 10.1103/PhysRevLett.95.237601

Introduction. - Organic molecules with strong electron accepting character, such as tetracyanoquinodimethane (TCNQ) and its derivatives, have been studied extensively because unique properties result when these molecules are combined with other materials. For example, charge transfer organic crystals are formed in conjunction with electron donating molecules, which exhibit metal-insulator transitions [1], exceptionally high conductivity [2-4], and even superconductivity $[5,6]$. (Organo)metal complexes of TCNQ posses electrical bistability [7,8], which renders them potential candidates for memory devices. More recently, the fluorinated TCNQ-analog 2,3,5,6-tetrafluoro7,7,8,8-tetracyanoquinodimethane (F4-TCNQ) has received considerable attention. It significantly improved device performance $[9,10]$ when coevaporated with electron donor molecular species to form hole transport layers in organic light emitting devices. This was attributed to the reduction of the hole-injection barrier $\left(\Delta_{h}\right)$ due to the formation of a narrow space-charge region (several $\mathrm{nm}$ ) near the metal contact, facilitated by charge transfer between F4-TCNQ and the organic donor matrix [11-13]. The conductivity in thin doped films increased by several orders of magnitude, both for crystalline (vanadyl- [14] and zincphthalocyanine; $\mathrm{ZnPc}[11,15])$ and amorphous [ZnPc [15], and N, $\mathrm{N}^{\prime}$-bis-(1-naphthyl)-N, $\mathrm{N}^{\prime}$-diphenyl-1,1-biphenyl4,4'-diamine; $\alpha$-NPD [13]] samples. Modifications of the metal electrode by the acceptor and the resulting changes in the energy-level alignment have not yet been investigated. Another important issue is the space-charge region formation mechanism in doped organic systems in the absence of an electric field and the associated energy-level bending [11-13]. In organic materials, charge carriers are highly localized due to polaronic effects $[16,17]$. Hence, the assumption of "free" charge carriers that can overcome the mutual Coulomb attraction in doped systems is, particularly in disordered systems, no longer valid [18].
PACS numbers: 79.60.Fr, 73.20.Hb, 73.61.Ph, 79.60.Jv

The present experiments were designed to highlight the importance of the acceptor-metal interaction on the energy-level alignment at organic-metal interfaces. We demonstrate that the acceptor (F4-TCNQ) reacts with $\mathrm{Au}$ surfaces, and that the energy levels of subsequently deposited organic materials can be pinned right at the interface. By comparing organic materials that form charge transfer complexes with F4-TCNQ [ $\alpha$-sexithienyl (6T) and $\alpha$-NPD] with one that does not ( $p$-sexiphenyl; 6P), we can conclude that the pinning-behavior is independent of charge transfer complex formation between the organic molecules and the acceptor. Furthermore, we propose that the hole-injection barrier at virtually any organicmetal interface can be optimized by using an appropriate acceptor precoverage of the metal surface.

Experiment.-Photoemission experiments were performed at the end station SurICat (beam line PM4) at the synchrotron light source BESSY (Berlin, Germany) [19]. The ultrahigh vacuum system consists of interconnected sample preparation (base pressure: $1 \times 10^{-8}$ mbar) and analysis (base pressure: $1 \times 10^{-10} \mathrm{mbar}$ ) chambers. Excitation energy for ultraviolet photoelectron spectroscopy (UPS) was $32 \mathrm{eV}$; the spectra were collected with a hemispherical electron energy analyzer (Scienta SES 100) with $120 \mathrm{meV}$ energy resolution. For x-ray photoelectron spectroscopy (XPS), excitation energies were 630 and $800 \mathrm{eV}$ (50 eV pass energy). Additional UPS experiments were performed at the FLIPPER II beam line at HASYLAB (Hamburg, Germany) [20,21]. Au substrates were prepared by vacuum depositing the metal in situ on a $\mathrm{Cu}$-foil and $\mathrm{Ar}-$ ion sputtering. F4-TCNQ (Aldrich), 6P (TCI Europe), $\alpha$-NPD (Aldrich), and 6T (Aldrich) were evaporated from resistively heated pinhole sources. Film mass thicknesses were monitored with a quartz crystal microbalance. F4-TCNQ was evaporated in a separate preparation chamber (pressure $<2 \times 10^{-9}$ mbar) to avoid cross contamina- 
tion of the other organic materials. Photoelectron spectra were recorded with a double-pass cylindrical mirror analyzer (resolution $150 \mathrm{meV}$ ) and a photon energy of $22 \mathrm{eV}$. The secondary electron cutoff (for determination of sample work function or ionization energy) was measured with $-3 \mathrm{~V}$ sample bias. Fitting of XPS spectra (Gaussian peaks and Shirley background) was performed with the program WINSPEC (Namur University).

Results and discussion.-Photoemission spectra of polycrystalline $\mathrm{Au}$ before and after deposition of nominally $5 \AA$ and $60 \AA$ F4-TCNQ are shown in Fig. 1(a). An overall attenuation of Au features, accompanied by the emergence of a new photoemission peak near $9 \mathrm{eV}$ binding energy (BE), can be seen. The sample work function $(\phi)$ increased from 5.25 (pristine $\mathrm{Au}$ ) to $5.6 \mathrm{eV}$ (5 A F4-TCNQ) as revealed by the shift of the secondary electron cutoff shown in Fig. 1(b). After $5 \AA$ F4-TCNQ deposition the close-up of the region near the Fermi level in Fig. 1(c) has additional intensity. This is indicated by the shaded areas centered at 0.45 and $1.45 \mathrm{eV}$, according to the difference spectrum in Fig. 1(c) where the Au contribution was subtracted. XPS measurements for $5 \AA$ F4-TCNQ/Au [Fig. 1(d)] showed two peaks in the $N 1 s$ region at 397.8 and $399.15 \mathrm{eV} \mathrm{BE}$, both $\pm 0.05 \mathrm{eV}$. Further deposition of
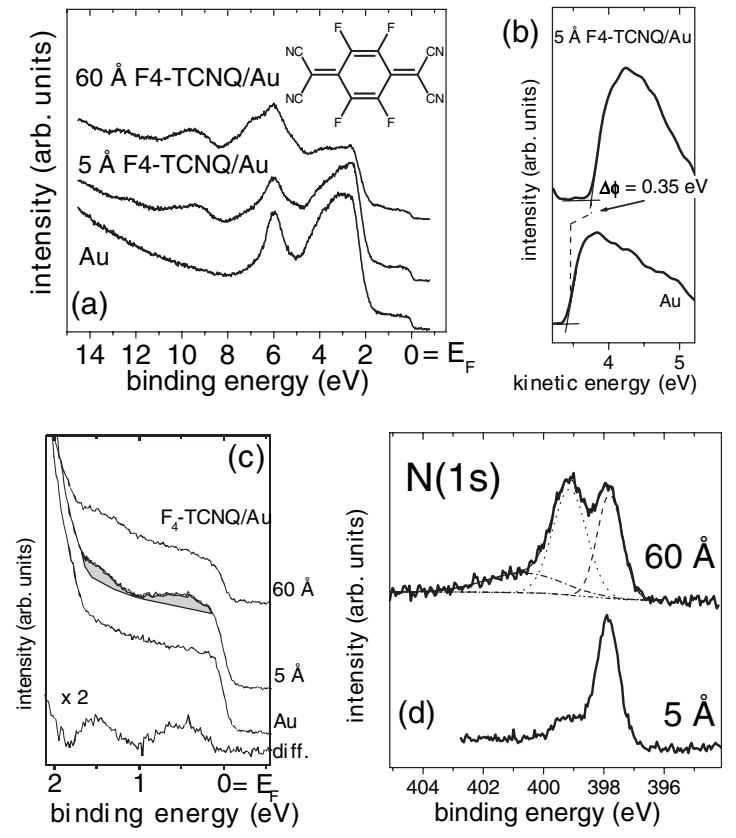

FIG. 1. (a) UPS spectra (photon energy, $32 \mathrm{eV}$ ) of pristine polycrystalline $\mathrm{Au}$ and after deposition of 5 and 60 A F4-TCNQ (top). Inset: chemical structure of F4-TCNQ. (b) Secondary electron cutoff region (at sample bias $-3 \mathrm{~V}$ ) for pristine $\mathrm{Au}$ (bottom) and $5 \AA$ F4-TCNQ/Au (top), indicating the work function change $\Delta \phi$. (c) Near the $E_{F}$ region of spectra from part (a); the shaded area indicates the new photoemission features induced by F4-TCNQ. Bottom spectrum is the difference between $5 \AA \mathrm{F} 4-\mathrm{TCNQ} / \mathrm{Au}$ and pristine $\mathrm{Au}$ (scaled to the intensity at $E_{F}$ ). (d) $N$ is XPS spectra (photon energy, $630 \mathrm{eV}$ ) of $5 \AA$ F4-TCNQ/Au, and $60 \AA$ F4-TCNQ/Au (with peak components and background obtained by the fitting routine).
F4-TCNQ (60 $\AA$ ) led to an intensity increase in the higher BE component in the $N 1 s$ spectrum. Scanning tunneling microscopy experiments on F4-TCNQ on $\mathrm{Au}(111)$ [22] confirm that the acceptor molecules remain intact when adsorbed on Au. We propose a significant charge transfer between $\mathrm{Au}$ and F4-TCNQ. In analogy to the XPS results reported for the unfluorinated parent molecule TCNQ [23,24], we assign the higher BE $N(1 s)$ peak to neutral F4-TCNQ (in multilayers) and the lower BE peak to the anion species (in direct contact with $\mathrm{Au}$ ). The third broad component centered at $400.5 \pm 0.05 \mathrm{eV}$ BE is attributed to shake-up processes [23,24]. The proposed electron transfer is fully consistent with the appearance of two new peaks close to $E_{F}$ in the UPS spectra [Fig. 1(c)] [21]. These peaks are derived from the relaxed highest occupied molecular orbital (HOMO; higher BE) and the stabilized - now (partially) filled-lowest unoccupied molecular orbital (LUMO; lower BE) [17,21] of the pristine molecule. After deposition of F4-TCNQ multilayers, in which the molecules are in their neutral state (as also suggested by XPS results), the intensity of these two peaks is reduced [top spectrum in Fig. 1(c)]. The work function increase after F4-TCNQ deposition indicates electron transfer from $\mathrm{Au}$ to the organic molecule.

Having established that F4-TCNQ forms a charge transfer complex on the surface of $\mathrm{Au}$, we now turn to the influence of different F4-TCNQ precoverages $\left(\theta_{\mathrm{F} 4-\mathrm{TCNQ}}\right)$ on the interface energetics when another organic molecular material is deposited afterwards. The first material we investigated was $\alpha$-sexithienyl (6T). Charge transfer complex formation between oligothienylenes and F4-TCNQ is known $[25,26]$; therefore, this material pair is of direct relevance for testing the concept of energy level bending within the framework of organic electrical doping [11-13]. UPS spectra for increasing 6T coverage on Au with $0.15 \AA$ F4-TCNQ preadsorbed exhibit a systematic trend [Fig. 2(a)] frequently observed in photoemission studies of organic-metal interfaces $[27,28]$. After $5 \AA 6 \mathrm{~T}$ deposition, the signal from the substrate was attenuated $(0-$ $0.65 \mathrm{eV}$ region), and a prominent new photoemission feature arises centered at $1.1 \mathrm{eV}$ originating from the HOMO of 6T. Subsequent 6T deposition resulted in a sharpening of the $6 \mathrm{~T}$ features, and a rigid shift towards higher BE by about $0.15 \mathrm{eV}$. Notably, the hole-injection barrier $\left(\Delta_{\mathrm{h}}\right.$; defined as the energy difference between $E_{F}$ and the linear extrapolation of the HOMO low BE leading edge) increased from 0.65 (monolayer range) to $0.80 \mathrm{eV}$ (nominally $150 \AA 6 \mathrm{~T}$ ). Shifts of this kind and magnitude can be attributed to decreased photohole screening efficiency for molecules at larger distances from the metal surface, consistent with earlier reports on this effect [27,28]. For comparison, the dashed curve in Fig. 2(a) shows the UPS spectrum of a nominally $150 \AA$ thick $6 \mathrm{~T}$ film on pristine $\mathrm{Au}$, where $\Delta_{\mathrm{h}}$ was $0.95 \mathrm{eV}$. Figure 2(b) provides direct evidence that higher $\theta_{\mathrm{F} 4-\mathrm{TCNQ}}$ resulted in a decrease of $\Delta_{\mathrm{h}}$ for $6 \mathrm{~T}$. For a $100 \AA$ thick $6 \mathrm{~T}$ film and $\theta_{\mathrm{F} 4-\mathrm{TCNQ}}=5 \AA$ we 

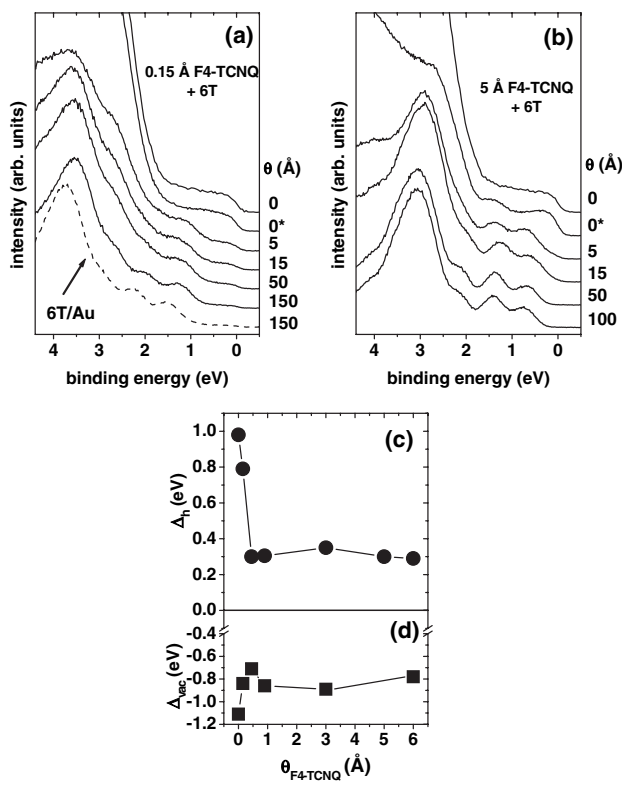

FIG. 2. (a) UPS spectra (photon energy, $22 \mathrm{eV}$ ) for increasing 6T coverage $\theta$ of $0.15 \AA$ F4-TCNQ precovered Au. Dashed spectrum: 6T on pristine $\mathrm{Au}$. " 0 " denotes the pristine $\mathrm{Au}$, " 0 *" F4-TCNQ precovered Au. (b) UPS spectra (photon energy, $22 \mathrm{eV}$ ) for increasing 6T coverage $\theta$ of $5 \AA$ F4-TCNQ precovered Au. " 0 " denotes the pristine Au, " 0 *" F4-TCNQ precovered Au. (c) Hole-injection barrier $\Delta_{\mathrm{h}}$ and (d) vacuum-level shift relative to pristine $\mathrm{Au} \Delta_{\text {vac }}$ for $6 \mathrm{~T}$ (at $100 \AA$ thickness) as function of F4-TCNQ precoverage $\theta_{\mathrm{F} 4-\mathrm{TCNQ}}$. The symbol size corresponds to the experimental error.

measured $\Delta_{\mathrm{h}}=0.3 \mathrm{eV}$ [Fig. 2(c)]. Values for the vacuumlevel shift (relative to the pristine Au surface, i.e., sample work function change) $\Delta_{\text {vac }}$ are shown in Fig. 2(d).

To elucidate whether charge transfer between F4-TCNQ and the subsequently deposited organic material is the dominant mechanism for the observed lowering of $\Delta_{\mathrm{h}}$, we have performed corresponding experiments on $p$-sexiphenyl (6P) [Fig. 3(a)]. Charge transfer can be ruled out in this case because of the large ionization energy of 6P of around $5.9 \mathrm{eV}[21,27,29]$, whereas the electron affinity of F4-TCNQ is only $5.24 \mathrm{eV}$ [13]. However, $\Delta_{\mathrm{h}}$ was significantly reduced for 6P when F4-TCNQ was preadsorbed on $\mathrm{Au}$. The minimum $\Delta_{\mathrm{h}}=0.6 \mathrm{eV}$ was achieved with only $1.2 \AA$ F4-TCNQ/Au [Fig. 3(b)]. Therefore, the huge reduction of $\Delta_{\mathrm{h}}$ (for comparison, $1.8 \mathrm{eV}$ for $6 \mathrm{P}$ on pristine $\mathrm{Au}$ [21]) by $1.2 \mathrm{eV}$ was not due to the formation of an organic charge transfer complex, or electrical doping, but was simply due to the modification of the Au surface by the strong electron acceptor F4-TCNQ, shown schematically in Fig. 3(c).

Results from a third example ( $\alpha$-NPD) are summarized in Fig. 3(d). This can be seen as an intermediate case, where weaker charge transfer between the two organic materials has been claimed [13], due to the similarity of $\alpha$-NPD ionization energy (5.3 eV [13,28]) and F4-TCNQ electron affinity $(5.24 \mathrm{eV})$. Nevertheless, the dependence
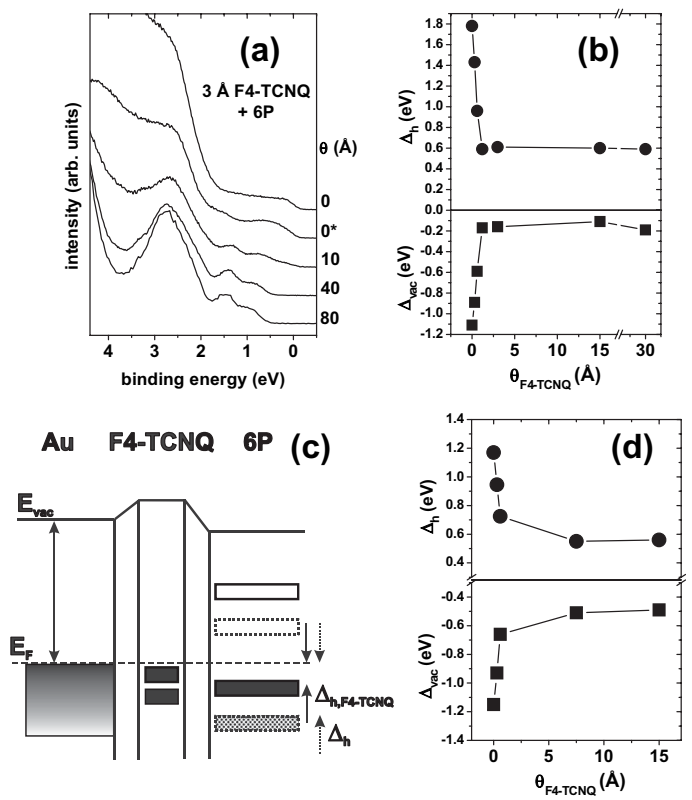

FIG. 3. (a) UPS spectra (photon energy, $22 \mathrm{eV}$ ) for increasing 6P coverage $\theta$ of $3 \AA$ F4-TCNQ precovered Au. " 0 " denotes the pristine $\mathrm{Au}$, " 0 *" F4-TCNQ precovered $\mathrm{Au}$. (b) Hole-injection barrier $\Delta_{\mathrm{h}}$ and vacuum-level shift relative to pristine $\mathrm{Au} \Delta_{\mathrm{vac}}$ as function of F4-TCNQ precoverage $\theta_{\mathrm{F} 4-\mathrm{TCNQ}}$ for $6 \mathrm{P}$ (at $80 \AA$ thickness). The symbol size corresponds to the experimental error. (c) Schematic energy-level diagram of $6 \mathrm{P}$ on $\mathrm{Au}$ with preadsorbed F4-TCNQ. F4-TCNQ region: black bars indicate (filled) LUMO- and HOMO-derived features of F4-TCNQ after electron transfer from Au. 6P region: HOMO (black bar) and LUMO (white bar) and hole-injection barrier $\Delta_{\mathrm{h}, \mathrm{F} 4-\mathrm{TCNQ}}$, and corresponding dotted symbols for $\theta_{\mathrm{F} 4-\mathrm{TCNQ}}=0 \AA\left(\Delta_{\mathrm{h}}\right)$. (d) As in (b) but for $\alpha$-NPD (at $50 \AA$ thickness).

of $\Delta_{\mathrm{h}}$ on $\theta_{\mathrm{F} 4-\mathrm{TCNQ}}$ follows the same trend as for 6P. $\Delta_{\mathrm{h}}$ decreases from 1.2 (pristine $\mathrm{Au}$ ) to $0.55 \mathrm{eV}(>0.6 \AA$ F4-TCNQ) for $\alpha$-NPD films of $50 \AA$ thickness. This is in between the values of $0.62 \mathrm{eV}$ for $0.5 \%$ - and $0.36 \mathrm{eV}$ for $30 \%$-F4-TCNQ-doped $\alpha$-NPD [13]. We also measured $\Delta_{\mathrm{h}}=0.35 \mathrm{eV}$ for thin $\alpha$-NPD films $(<10 \AA)$ for $\theta_{\mathrm{F} 4-\mathrm{TCNQ}}>7.5 \AA$ (spectra not shown).

We conclude that the energy-level alignment at organicmetal interfaces in the presence of a strong electron acceptor is largely determined by the number of acceptor molecules in direct contact to the metal substrate. If charge transfer between the acceptor and the subsequently deposited organic material can be excluded, acceptor precoverage is the only parameter determining $\Delta_{\mathrm{h}}$. Once the energylevel alignment is defined at the interface, no further shifts of energy levels as a function of film thickness occur [cf. Fig. 3(c), except for photohole screening effects]. The consequence is that the observation of energy level bending in coevaporated samples (i.e., doping) probably arises from more acceptor molecules reaching the metal surface as the film thickness is increased. The possibility of extensive F4-TCNQ diffusion through an organic matrix has been reported [13]; however, since most organic molecular 
materials do not follow a layer-by-layer growth mode [30], increasing the film thickness will also increase the amount of acceptor at the metal surface. In fact, we investigated the effect of coevaporation of 6T and F4-TCNQ, and found very similar energy-level shifts as a function of film thickness as those reported for Zn-Pc [11,12] and $\alpha$-NPD [13] coevaporated with F4-TCNQ.

Our key result is that, for all three investigated material pairs, $\Delta_{\mathrm{h}}$ decreased with increasing F4-TCNQ precoverage (on $\mathrm{Au}$ ) until a "critical coverage" $\left(\theta_{\text {crit }}\right)$ was reached, and remained virtually constant for larger $\theta_{\mathrm{F} 4-\mathrm{TCNQ}}$. In particular, for 6P [Fig. 3(b)] and $\alpha$-NPD [Fig. 3(d)] it appears that in the range of $\theta_{\mathrm{F} 4-\mathrm{TCNO}}=0, \ldots, 0_{\text {crit }}$ a linear relationship may exist between $\Delta_{\mathrm{h}}$ and $\theta_{\mathrm{F} 4-\mathrm{TCNQ}}$. The molecular levels of F4-TCNQ are pinned relative to the Au substrate by a charge transfer-type interaction. However, for submonolayer coverage, the surface potential felt by subsequently deposited molecules is an average of local $\phi$ 's of covered and uncovered substrate patches [31-34]. The energy levels of the second material are then aligned relative to the coverage-dependent, area-averaged surface potential established by F4-TCNQ/Au [33]. This is supported by our observation that the width of photoemission features from 6T, 6P, and $\alpha$-NPD did not depend on $\theta_{\mathrm{F} 4-\mathrm{TCNQ}}$, which would be the case if the spectra arose from the superposition of emission from patches with underlying bare Au and those precovered with F4-TCNQ.

This mechanism undoubtedly holds for material pairs without charge transfer. The situation becomes more complex, if the second organic material has electron donor-type character: molecules may react with F4-TCNQ that is not in contact with $\mathrm{Au}$ (multilayer), or may even interact strongly with Au-F4-TCNQ, resulting in a charge transfer complex with three components. This can explain the unexpected behavior of $\Delta_{\mathrm{vac}}$ for 6T and F4-TCNQ [Fig. 2(d)]: while $\Delta_{\mathrm{h}}$ was reduced by $0.7 \mathrm{eV}, \Delta_{\mathrm{vac}}$ changed by only $0.3-0.4 \mathrm{eV}$. In contrast, these two values change by the same amount for 6P and $\alpha$-NPD [Figs. 3(b) and 3(d)], corresponding to a rigid shift of all energy levels (including the vacuum level) as function of $\theta_{\mathrm{F} 4-\mathrm{TCNQ}}$.

Conclusion. - Our photoemission data provide direct evidence for a charge transfer-type interaction of F4TCNQ with Au surfaces. The hole-injection barriers at interfaces between F4-TCNQ/Au and three electroactive conjugated organic materials (6T, 6P, and $\alpha$-NPD) were measured as a function of F4-TCNQ precoverage. Significant reduction of $\Delta_{\mathrm{h}}(0.65 \mathrm{eV}$ for 6T and $\alpha-\mathrm{NPD}$, $1.2 \mathrm{eV}$ for $6 \mathrm{P}$ ) was found in all three cases, independent of charge transfer complex formation between the conjugated organic materials and F4-TCNQ. Furthermore, $\Delta_{\mathrm{h}}$ could be tuned systematically over a wide range by appropriate adjustment of $\theta_{\mathrm{F} 4-\mathrm{TCNQ}}$. This is explained in terms of the $\theta_{\mathrm{F} 4-\mathrm{TCNQ}}$ dependence of the area-averaged surface potential of F4-TCNQ/Au substrates. Our results suggest that by using (sub)monolayer coverages of strong electron acceptors on metal surfaces the hole-injection barriers at virtually any organic-metal interface can be optimized.
The authors thank Hendrik Glowatzki for assistance during photoemission experiments. N. K. acknowledges financial support from the Emmy Noether Program of the DFG.

*Electronic address: norbert.koch@physik.hu-berlin.de

[1] C. W. Chu et al., Phys. Rev. Lett. 31, 1491 (1973).

[2] R. G. Kepler, P.E. Bierstedt, and R.E. Merrifield, Phys. Rev. Lett. 5, 503 (1960).

[3] J. Ferraris et al., J. Am. Chem. Soc. 95, 948 (1973).

[4] R. Kumai, Y. Okimoto, and Y. Tokura, Science 284, 1645 (1999).

[5] L. B. Coleman et al., Solid State Commun. 12, 1125 (1973).

[6] H. Seo, C. Hotta, and H. Fukuyama, Chem. Rev. 104, 5005 (2004).

[7] R. S. Potember, T. O. Poehler, and D. O. Cowan, Appl. Phys. Lett. 34, 405 (1979).

[8] T. Reda et al., J. Phys. Chem. B 107, 13774 (2003).

[9] J. Blochwitz et al., Appl. Phys. Lett. 73, 729 (1998).

[10] X. Zhou et al., Appl. Phys. Lett. 78, 410 (2001).

[11] W. Y. Gao and A. Kahn, Appl. Phys. Lett. 79, 4040 (2001).

[12] J. Blochwitz et al., Organic Electronics 2, 97 (2001).

[13] W. Y. Gao and A. Kahn, J. Appl. Phys. 94, 359 (2003).

[14] M. Pfeiffer, A. Beyer, T. Fritz, and K. Leo, Appl. Phys. Lett. 73, 3202 (1998).

[15] B. Maennig et al., Phys. Rev. B 64, 195208 (2001).

[16] E. A. Silinsh, Organic Molecular Crystals (Springer, Berlin, 1980), Vol. 16.

[17] W. R. Salaneck, R. H. Friend, and J. L. Bredas, Phys. Rep. 319, 231 (1999).

[18] M. Brinkmann et al., J. Phys. Chem. A 108, 8170 (2004).

[19] A. Vollmer et al., Eur. Phys. J. E 17, 339 (2005).

[20] R. L. Johnson and J. Reichardt, Nucl. Instrum. Methods Phys. Res. 208, 791 (1983).

[21] N. Koch et al., J. Phys. Chem. B 104, 1434 (2000).

[22] F. Jäckel, Doctoral thesis, Humboldt-Universität zu Berlin, 2005.

[23] J. M. Lindquist and J. C. Hemminger, J. Phys. Chem. 92, 1394 (1988).

[24] C. Rojas, J. Caro, M. Grioni, and J. Fraxedas, Surf. Sci. 482, 546 (2001).

[25] S. Hotta and K. Waragai, J. Mater. Chem. 1, 835 (1991).

[26] G. Brocks, Phys. Rev. B 55, 6816 (1997).

[27] H. Ishii, K. Sugiyama, E. Ito, and K. Seki, Adv. Mater. 11, 605 (1999).

[28] A. Kahn, N. Koch, and W. Y. Gao, J. Polym. Sci., B, Polym. Phys. 41, 2529 (2003).

[29] N. Koch et al., Adv. Funct. Mater. 11, 51 (2001).

[30] G. Witte and C. Wöll, J. Mater. Res. 19, 1889 (2004).

[31] N.D. Lang and A. R. Williams, Phys. Rev. B 25, 2940 (1982).

[32] R. Fischer et al., Phys. Rev. Lett. 70, 654 (1993).

[33] G. Koller, F. P. Netzer, and M. G. Ramsey, Appl. Phys. Lett. 83, 563 (2003).

[34] Photoemission in Solids I, Topics in Applied Physics Vol. 26, edited by M. Cardona and L. Ley (Springer, Berlin 1978), p. 16. 